\title{
Sharing Multi-User VR Spaces
}

\author{
Morgan C. Evans* \\ Srujani Kamineni* \\ morganev@cs.cmu.edu \\ kamineni.srujani@gmail.com \\ Carnegie Mellon University \\ Shan Jiang \\ shanj@andrew.cmu.edu \\ Carnegie Mellon University
}

\author{
Omar Cheikh-Ali \\ osc@andrew.cmu.edu \\ Carnegie Mellon University
}

\author{
Justin Fanzo \\ jfanzo@alumni.cmu.edu \\ Carnegie Mellon University
}

\author{
Miao Ren \\ mren1@alumni.cmu.edu \\ Carnegie Mellon University
}

\author{
Ketul Majmudar \\ majmudarketul1@gmail.com \\ Carnegie Mellon University \\ Jessica Hammer \\ hammerj@andrew.cmu.edu \\ Carnegie Mellon University
}

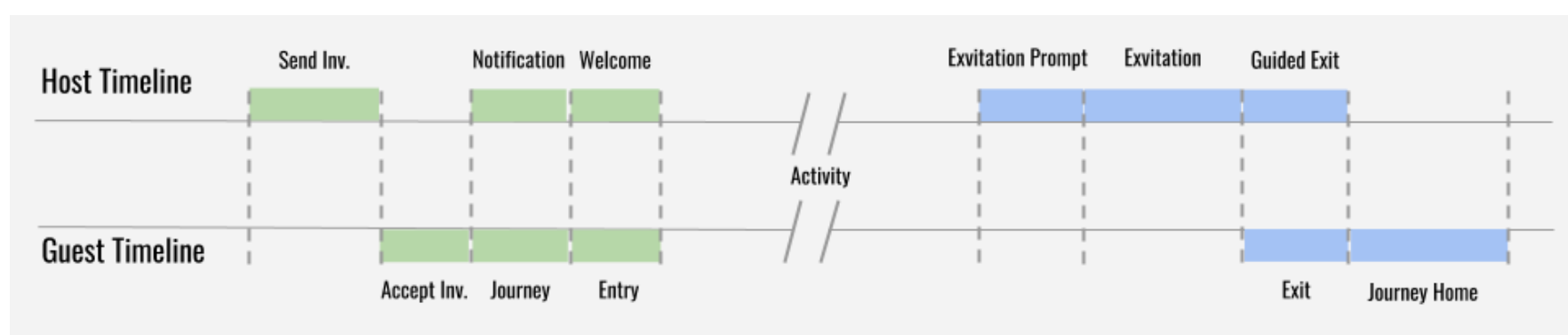

Figure 1: User experience timeline

\begin{abstract}
In shared virtual reality (VR) spaces, users can appear and disappear without transitions, in a pop-in, pop-out style. This solution to entry and exit might be an acceptable solution for certain settings, but produces uncertainty in more intimate settings. Our research explores alternate entry and exit paradigms for shared VR spaces. Based on anthropological studies of entry and exit rituals in physical homes, we created a user journey map for entry and exit that differentiates the roles of host and guest in the shared VR space. We iteratively developed Unity prototypes that allowed a "host" to invite a "guest" into their virtual space, engage them in simple interactions, and invite the guest to depart. We define exvitation as a critical stage in the guest's departure-a social permission for a guest to depart the host's home. We identify exvitation prompts that were salient to our users and report of how these prompts were received by participants in our playtests. Future work on our
\end{abstract}

${ }^{*}$ Both authors contributed equally to this research.

Permission to make digital or hard copies of part or all of this work for personal or classroom use is granted without fee provided that copies are not made or distributed for profit or commercial advantage and that copies bear this notice and the full citation on the first page. Copyrights for third-party components of this work must be honored

For all other uses, contact the owner/author(s).

CHI PLAY '20 EA, November 2-4, 2020, Virtual Event, Canada

(C) 2020 Copyright held by the owner/author(s).

ACM ISBN 978-1-4503-7587-0/20/11.

https://doi.org/10.1145/3383668.3419871 user journey map as well as our exvitation prompts can aid in the expansion of playful experiences possible in VR.

\section{CCS CONCEPTS}

- Human-centered computing $\rightarrow$ Virtual reality.

\section{KEYWORDS}

Virtual reality; augmented home; rituals; invitation; exvitation

ACM Reference Format:

Morgan C. Evans, Srujani Kamineni, Omar Cheikh-Ali, Justin Fanzo, Shan Jiang, Ketul Majmudar, Miao Ren, and Jessica Hammer. 2020. Sharing MultiUser VR Spaces. In Extended Abstracts of the 2020 Annual Symposium on Computer-Human Interaction in Play (CHI PLAY '20 EA), November 2-4, 2020, Virtual Event, Canada. ACM, New York, NY, USA, 5 pages. https: //doi.org/10.1145/3383668.3419871

\section{INTRODUCTION}

In physical spaces, entry and exit are socially complicated interactions that people use physical and social cues to manage. For example, a store manager makes an announcement that the store will be closing in 30 minutes, inviting guests to conclude their shopping. The announcement communicates a boundary that exists in brick and mortar stores, namely that there are store hours. In the context of a home space, there are no such guidelines for open and closed hours so entry and exit must be somehow determined by at least one of the social roles. 
VR offers similar issues about entry and exit. Many VR systems have "owners" of a space where other users are "guests" who can enter and exit. However, existing systems do not support interactions and rituals. Current design for entry and exit is pop-in and pop-out, which is the abrupt appearing and disappearing of a user. The instantaneous arrival of guests in a VR home space redefines the original sentiment of being at home, and not positively.

If we figure out how to navigate entry and exit rituals effectively for VR spaces, we can begin to create and explore new types of social relationships. Users may find comfort and familiarity towards the virtual world through these relationships-engaging in many social dynamics that may directly translate from the real world, like laughing at a joke. This type of social atmosphere can create new affordances for shared VR play, such as games that require intimacy or "cozy" games.

In this paper, we propose a user journey for invitation in VR, and its partner activity, "exvitation," or the socially complicated process of departure. The contributions of this paper are two-fold: we formalize the shared experience timeline of the guest and host using iterative design, and using the timeline we create a prototype to test exvitation triggers called exvitation prompts. The following sections include the creation of the user journey map, a description of the map stages, followed by our playtests and preliminary findings, and future work.

\section{BACKGROUND}

Rituals about the entry and exit of a home communicate privacy principles and reinforce social dynamics between a host and a guest. Després describes meanings of what the home embodies, including feelings of privacy, security, and ownership [2]. The door, a threshold, designates the transition between the public and private space, controlled by the host as the owner of the home. An open door in 1700s England was regarded as an invitation, only after sunset was it ever locked [9]. Such entry rituals serve as one example of what Desjardins et al. categorize as social routines in HCI research [1]. Such an interconnected web of social routines create a home, and need to be understood before the addition of interactive technologies [1].

Our research seeks to understand social dynamics around hosting and guesting in multi-user VR. While the "moment of exit" from the virtual back into the physical world has been studied for effects on perception [5], how events unfold before this transition, especially in shared social settings, remains largely unexplored. However, multi-user VR has been studied in a number of other settings including serious games such as paramedic vocational training, where the collaborative and stressful environment relies on the intuitiveness of the virtual environment to make decisions, like user inventory design $[7,10]$. Learning in VR for real world experience relies on user's ability to translate facts from the real world to the virtual world. In addition to games, Roth et al. studied eye contact signifiers and concepts like joint attention and grouping as metrics for social behaviors in a multi-user VR museum environment [6]. Finally, some co-located multi-user VR systems allow one player to use the immersive controllers while the other engages through an external display [3].
In this paper we address invitation and exvitation in a virtual home. A breadth of virtual homes exist from augmented reality (AR) to substitutional reality [8], where objects in the physical world map directly to objects in the virtual world. Hericks et al. designed three virtual rooms and tested their ability for users to translate their attachment from their physical homes to their virtual homes[4].

\section{MAP AND SYSTEM DESIGN}

To create our user map, we began by exploring the timeline of activities for people visiting a friend in person. We interviewed 18 student participants, asking them to describe in detail a time when they paid a visit and/or hosted a friend. Questions focused on the process of invitation and entry, and on the process of departure. Participants also reported the central activities of the visit, including socializing (18/18), drinking (12/18), and cooking (9/18).

For each interview, we reconstructed the visit in temporal order and divided them by host and guest activities. We then coded activities into phases, described below. Our phase codes were developed based on common activities across all 18 interviews, and refined in consultation with an anthropologist who served as an expert advisor. For example, the "Notification" phase often involved the guest texting to inform the host that they are on their way, while the guest was in transit ("Journey").

We then tested our map against existing multi-user VR experiences. We randomly sampled three experiences from a set of multi-user VR systems that allow one user to visit another in their virtual space. For each selected experience, we watched one or more user testing videos of users visiting one another. We noted whether each stage of our journey map was present, and how each stage was supported (or not supported) by the existing technology. We paid particular attention to moments of awkwardness or unease, where the technological tools available did not seem to meet the users' social needs. Finally, we watched for any behaviors around entry and exit that were not captured by our prior codes. At this stage, we did not observe novel behaviors and therefore did not add any new codes; however, these observations helped us formalize the notion of exvitation, described below.

We used our map to create a digital system in Unity, so that we could explore our insights through design. This system allowed for two synchronous users situated in two separate labs, one in the host role and one in the guest role. They could not hear or see each other. Each user had a private space to which only they had access. The guest began in an airy seaside room, while the host could be placed either in an intimate and cozy room (the Hearth) or a wideopen yard (the Garden). The system implements the map stages, described below, in allowing the guest to visit the host. During the visit, the host and guest had access to real-time voice chat and synchronous shared-state experiences in the host's space.

\section{STAGE DESCRIPTION}

The user journey map (Figure 1) shows the coordination process for an activity that two people experience in a shared virtual environment. One user is the host, in whose space the shared experience takes place. The other is the guest, who is a temporary visitor in that space. Figure 1 is horizontally aligned, with the host timeline 
above the guest timeline; both timelines run chronologically from left to right.

\subsection{Invitation}

While our findings for the invitation process align with prior work on the topic, we share the stages and examples of how they manifested in practice.

Send invitation. An invitation is typically initiated by the host, although guests in physical spaces occasionally reported inviting themselves over. As both users may have private spaces to which they could invite the other, this stage defines which user is the host (owner of the space) and which user is the guest (visitor in the space). In our system, the host uses a virtual display console to select from a list of friends. After selecting a friend and confirming that they want to invite that friend over, an invitation is issued.

Accept invitation. The guest receives the host's invitation and agrees to the visit. In physical spaces, this is typically a social negotiation; in virtual spaces, where access to another person's space needs to be explicitly granted, the guest must indicate their official acceptance in a way the system understands. In our system, the guest receives a message on their own virtual console with details of the invitation. If they click the "Accept" button, they have accepted the invitation.

Notification. Both guests and hosts reported needing to understand when and how the guest would appear in the host's space. For example, guests in VR spaces who suddenly appeared without warning would cause considerable social discomfort for both parties. In our system, we explicitly designed journeys that took some time (see next stage). The host was automatically notified on their console when their guest was in transit. Meanwhile, the host could pursue other activities in their virtual space.

fourney. In physical spaces, the process of moving from one person's space to another takes some time. In VR environments, an abrupt transition into someone else's space was a common source of discomfort, producing uncomfortable laughter. In our system, we therefore designed a set of slow transitions from one VR space to the other. This decision gave the host time to prepare (see above) and provided a liminal space where the guest could transition to their new social role. For example, one slow transition had the guest descend into the host's space in a hot-air balloon.

Welcome. The host indicates that the guest has permission to enter. This might have a physical manifestation such as opening the door for the guest, or a social one such as welcoming them in a cheerful voice. In our system, the host takes control of the final step of the guest's journey, such as by opening the hot-air balloon's basket, to convey their acceptance of the guest.

Entry. The guest physically or virtually moves into the host's territory. This occurs after they have permission to pass. In VR environments, we observed that a lack of such permission created discomfort, such as when a guest put their head through the wall before being welcomed. In our system, the guest therefore could not move into the space until the welcome was complete. Only then could they enter.

\subsection{Activity}

The present work does not focus on the activities done by users jointly in the host's space. However, the activity contextualizes the guest's visit and provides a reason for them to enter and exit the host's space.

Based on our interviews, in which talking was the most common social activity, our system supports conversational activities such as trip planning or storytelling. It also includes simple VR interactive objects such as a throwable ball. However, the invitation and exvitation design could be applied to a wide range of activities such as two-player VR games.

\subsection{Exvitation}

While our mapping of the invitation process lined up with existing work on social invitations, we found that it was difficult even to describe the often-awkward process of having a guest depart. We therefore coin the term exvitation to describe this process. During exvitation, the host and guest come to the shared understanding that the shared activity is over and it is time for the guest to leave; the guest then departs and journeys home.

Exvitation prompt. How do people know when it is time to go home? In physical reality, people reported checking the time or being interrupted by outsiders. These incidents prompted the process of departure to begin. However, in VR these natural interruptions were typically not present. Starting the process of departure was extremely awkward. In our system, we therefore designed exvitation prompts, or environmental cues that can provide excuses to begin the process of departure.

Exvitation. Either the host or guest can indicate that it is time for the visit to end, typically referring to the exvitation prompt as an excuse. In VR spaces, this often ended in a confusing and awkward negotiation, as the users discussed the fact that the guest should leave without actually moving toward departure. In our system, the host initiates exvitation by indicating on their console that the visit has ended, similar to saying "Let me call you a car."

Guided exit. In many cases, we observed the host walking their guest to the exit - even in virtual reality spaces where the guest could simply disappear at any point. We interpret this choice as the host mitigating the awkwardness of departure and reassuring their guest that the relationship remains valued. In our system, the guest's departure point is shown within the space, so that the host can escort them there; the guest can only depart from that single spot, for example by entering the hot-air balloon's basket.

Exit. The guest departs the space. This typically involves crossing a boundary, such as stepping outside the door or closing the door of a virtual hot-air balloon. In our system, all exit methods involved a concrete and visible action that all parties knew would lead to the guest's removal from the space.

fourney home. The guest returns to their home space, while the host can go about their business. In our system, the journey home is a slow process, just as the inbound journey was. The transition gives the guest time to prepare for their arrival in a different social context, where they are the ones in charge of the space. 


\section{EXVITATION PROMPTS}

As a way of demonstrating the value of our model, we describe our work on "exvitation prompts" - cues that help hosts and/or guests initiate the conclusion of a visit.

Based on our interviews, we identified features of a successful exvitation prompt. First, it had to be noticeable by the right person. For example, if the guest's alarm went off but only the host noticed it, it made the host very uncomfortable to point it out. Second, it had to be appropriate. Exvitation prompts that were obviously fake were seen as potentially insulting. Finally, we noticed that initiation of the prompt mattered to users. Prompts that were environmental or automatic were much more comfortable than those that were initiated by the guest or host. To explore these themes, we designed four exvitation prompts: a candle burning down, a clock chiming, flowers blooming, and rain beginning.

Noticeable. The candle and the flowers did not interrupt the users' activity. They were only noticeable if someone looked at the appropriate area of the world and realized that something had changed. The clock and the rain both interrupted the user's activity with audio-visual cues.

Appropriate. The candle and the clock were both implemented in the Hearth space. They fit the space because the space already had a cozy fire and low lighting, as well as a large grandfather clock visible. The flowers and the rain were implemented in the Garden space, which was very green and open to the sky.

Initiation. For each prompt, we developed three methods through which it could be initiated. First, the researcher could initiate the prompt without any input from the participants. Second, the host could set a timer before the guest arrived; the prompt would happen when the timer ran out. Finally, the host could press a button, invisible to the guest, that would trigger the prompt immediately.

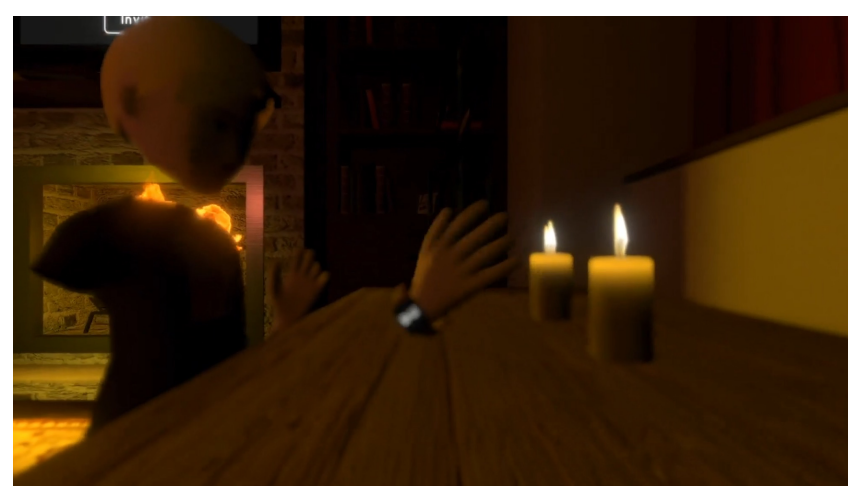

Figure 2: Candle burning down exvitation prompt

\section{PRELIMINARY FINDINGS}

Participants were recruited to playtest our exvitation prompts through fliers we distributed on local college campuses and public spaces. We recruited nine pairs of participants: four of the pairs were previously acquainted, while the five other pairs were paired randomly to simulate variety among the host and guest relationships. We used the HTC Vive and set up a room scale play area with physical furniture aligned with the virtual space. During the session, observational notes and recorded sessions of the virtual space were captured. Following test completion, we interviewed participants on topics of comfort in the interactions, sense of immersiveness, and comparisons with real life scenarios. We report preliminary findings related to the design features (discoverability, integration, and ownership) described above. In Figure 3, exvitation prompts are rated from subtle to disruptive based on our observations of playtests and interview responses.

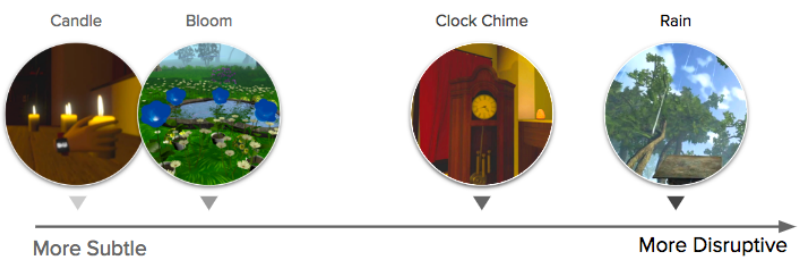

Figure 3: Subtle to disruptive exvitation prompts

Exvitation prompts provide an adequate excuse for hosts to invite the concept of departure, or for the guest to suggest it themselves. Both the candle and the flower cue were missed by some playtesters. While appropriate cues for the context in which they were used, the visual state change of the candle light (Fig 2) or the flower shape was not noticeable enough to trigger playtesters to suggest departure. Rain and bloom cues occurred over larger areas of the VR space and were more disruptive than the clock and candle, which presented at a physically smaller scale. Multiple playtesters responded to the rain cue by standing and immediately suggesting their departure. Their automatic response suggests the rain cue was highly appropriate and well integrated into the environment, even though no one was actually getting wet. Finally, we found that if playtesters missed the cue, they would initiate the exvitation using player-developed methods in real time. Examples include playtesters gifting interactable props to each other as parting gifts, or exchanging a high five before departure.

\section{FUTURE WORK}

Initial playtests on the exvitation prompt stage of the user journey map serve as a starting point to further explore social dynamics in virtual homes. Next steps include validating our exvitation prompt insights and testing other sections of the map, including the guest's journey to and from the shared virtual space. Finally, we plan to apply these design techniques directly to the creation of multi-user, synchronous VR games.

\section{ACKNOWLEDGMENTS}

We thank Lauren Herckis, Tom Corbett, and Heather Kelly. This research was funded by Oath.

\section{REFERENCES}

[1] Audrey Desjardins, Ron Wakkary, and William Odom. 2015. Investigating genres and perspectives in HCI research on the home. In Proceedings of the 33rd Annual ACM Conference on Human Factors in Computing Systems. 3073-3082.

[2] Carole Després. 1991. The meaning of home: Literature review and directions for future research and theoretical development. fournal of architectural and Planning Research (1991), 96-115. 
[3] Jan Gugenheimer, Evgeny Stemasov, Harpreet Sareen, and Enrico Rukzio. 2018 FaceDisplay: towards asymmetric multi-user interaction for nomadic virtual reality. In Proceedings of the 2018 CHI Conference on Human Factors in Computing Systems. 1-13.

[4] Lauren Herckis, Jessica Cao, Jacqui Fashimpaur, Anna Henson, Rachel Rodgers, Thomas W Corbett III, and Jessica Hammer. 2020. Exploring Hybrid VirtualPhysical Homes. In Proceedings of the 2020 ACM on Designing Interactive Systems Conference. 669-680.

[5] Jarrod Knibbe, Jonas Schjerlund, Mathias Petraeus, and Kasper Hornbæk. 2018 The dream is collapsing: the experience of exiting VR. In Proceedings of the 2018 CHI Conference on Human Factors in Computing Systems. 1-13.

[6] Daniel Roth, Constantin Klelnbeck, Tobias Feigl, Christopher Mutschler, and Marc Erich Latoschik. 2018. Beyond replication: Augmenting social behaviors in multi-user virtual realities. In 2018 IEEE Conference on Virtual Reality and $3 D$
User Interfaces (VR). IEEE, 215-222.

[7] Jonas Schild, Sebastian Misztal, Beniamin Roth, Leonard Flock, Thomas Luiz, Dieter Lerner, Markus Herkersdorf, Konstantin Weaner, Markus Neuberaer, Andreas Franke, et al. 2018. Applying multi-user virtual reality to collaborative medical training. In 2018 IEEE Conference on Virtual Reality and 3D User Interfaces (VR). IEEE, 775-776.

[8] Adalberto L Simeone and Eduardo Velloso. 2015. Substitutional reality: bringing virtual reality home. XRDS: Crossroads, The ACM Magazine for Students 22, 1 (2015), 24-29.

[9] Amanda Vickery. 2009. Behind closed doors. Yale University Press.

[10] Konstantin Wegner, Sven Seele, Helmut Buhler, Sebastian Misztal, Rainer Herpers, and Jonas Schild. 2017. Comparison of two inventory design concepts in a collaborative virtual reality serious game. In Extended Abstracts Publication of the Annual Symposium on Computer-Human Interaction in Play. 323-329. 